\title{
Applications of SIMS to cultural heritage studies
}

\author{
A. Adriaens ${ }^{a, *}$, M.G. Dowsett ${ }^{b}$

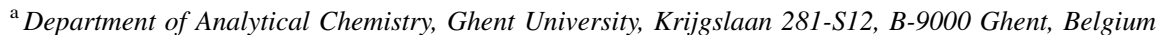 \\ ${ }^{\mathrm{b}}$ Department of Physics, University of Warwick, Warwick Coventry CV4 7AL, UK \\ Received 12 September 2005; accepted 15 February 2006 \\ Available online 30 June 2006
}

\begin{abstract}
This paper discusses the use of secondary ion mass spectrometry in cultural heritage studies recently reported in the literature, and includes material on the analysis of pigments, glass and metals. Applications of depth profiling, imaging, speciation and the topic of ultra-low energy SIMS depth profiling are addressed.
\end{abstract}

(C) 2006 Elsevier B.V. All rights reserved.

Keywords: SIMS; Cultural heritage; Inorganic materials; Imaging; Speciation; Depth profiling

\section{Introduction}

Europe has a particularly rich and diversified cultural heritage which both links its member countries and outlines their differences. It includes buildings, monuments and objects of all sizes. These are the fruits of both ancient and new technologies, and comprise a great variety of materials. Our heritage forms the basis of present and future cultures, and through the skills, beauty, and engineering genius it manifests, it is a vital source of inspiration and pleasure. Its exploitation, in the form of displayed objects, is a direct and indirect source of revenue through tourism, and at the same time the basis for a rich diversity of learning. The conservation of the European cultural heritage is for these reasons a culturally important activity in its own right, and an economic necessity.

Nevertheless, the physical part of the European cultural heritage is deteriorating faster than it can be conserved, restored or studied. Assets are being lost, or are at risk, through natural processes of decay (sometimes accelerated by poor environmental control), environmental disasters (sometimes exacerbated by human activity), the direct effects of enhanced public access (without commensurate conservation measures), conservation/preservation procedures whose long-term effects were and are not understood, simple negligence, looting and war $[1,2]$.

\footnotetext{
* Corresponding author. Tel.: +32 9264 4826; fax: +32 92644960.

E-mail address: annemie.adriaens@ugent.be (A. Adriaens).
}

The research required to alleviate this huge class of problems and at the same time progress the study of the European cultural heritage is of an interdisciplinary nature. This is because many different techniques are required in order to investigate and solve a specific problem. The analytical methods used in this field of research are identical with those used at the cutting edge of modern science [3-5]. Techniques developed for advanced physics, chemistry and biology (e.g. semiconductor, aerospace, medical and environmental research; forensic science, and biotechnology) have a commonality of application to both ancient and modern materials, since problems encountered in both the advanced technology and cultural heritage areas are similar. This is partly due to the heterogeneity of the materials involved at various scales of length. (It is also the case that new instrumentationnotably portable equipment-developed specifically for cultural heritage applications can be beneficially applied to advanced technology areas.) Using these techniques, we can help to establish authenticity and reach back through time to develop a deeper understanding of the craftsmanship and technology that was used. Such methods also allow us to study the alterations imposed on an artefact by time and then contribute to the development of adequate conservation treatments and simple diagnostic techniques for use in practical conservation.

However, there is one essential difference between the analysis of ancient and modern materials-an art object or ancient artefact cannot be replaced, and the consumption or damaging of even a small part of it for analytical purposes must be undertaken only where vital data cannot otherwise be 
obtained. Depending on the information required, one might use a combination of truly non-invasive techniques (i.e. those which do not require a sample to be removed from the object, and which leave the object in essentially the same state before and after analysis) and micro-destructive techniques (i.e. those which consume or damage a few picolitres of material and which may require the removal of a sample). In all cases, one should aim at the maximization of information and the minimization of the consumed volume. This brings us to the need for designed pathways which allow multi-instrument analytical approaches, including thought-through sample preparation schemes (removal from the object, preparation, and packaging for transport). Clearly established schemes lead to a better integration of activities and to an improvement of the relevance and the reproducibility of analytical results.

SIMS has contributed to a diverse range of problems including technology and authenticity, origin and provenance, degradation processes, such as corrosion and weathering, and conservation [6-9]. At first sight, the requirements of SIMS and of chemical analysis in the area of cultural heritage appear to be diametrically opposed. SIMS is a destructive analytical technique in the sense that sample material is consumed during the analysis (as with all forms of mass spectrometry). In addition, like many forms of ion beam analysis, electron microscopy and electron spectroscopies, the SIMS instrument can usually accommodate only a small sample with maximum linear dimensions of some mm. A larger artefact will need to be sampled in some way before SIMS analysis is possible. Finally, SIMS is a high or even ultra high vacuum technique, and depends on good vacuum over the sample for many aspects of its data quality. Therefore, the sample must be vacuum compatible. In fact, the latter requirement still allows samples as diverse as oil paint and obsidian to be analysed so this limitation is not so great. On the other hand, SIMS offers unique combinations of attributes which warrant its use under appropriate circumstances, including high depth and lateral resolution (nanometres and tens of nanometres, respectively), molecular specificity and fingerprinting and trace analysis in the ppm-ppb range. It is worth noting that although these specifications can undoubtedly be achieved individually, they are often mutually incompatible and even require different instruments for their attainment. Nevertheless, it is in combinations of properties that SIMS has its strengths, and also in complementary use with other techniques.

This paper provides an update to a previous paper by the authors [9] on recent applications in the field.

\section{Imaging of paints and pigments}

One of the major advantages of static SIMS resides in its potential to characterize and image both inorganic and organic analytes, thereby representing one of the few methods of closing the traditional gap between the fields of organic and inorganic analytical chemistry. This capability has been demonstrated in work by the research group at AMOLF [10-13] for the study of paint cross sections. Microscopic and molecular information about the nature and the distribution of pigments and binding media contributes to the answering of technical art historical questions, such as the deduction of the original appearance of the painting, and the establishment of the chemical and physical conditions before conservation and restoration [10]. The latter is essential when deciding upon the correct treatment method to conserve or restore the artefacts.

As an example Keune and Boon [11] studied a cross section taken from the Virgin's blue robe in the panel painting "The descent from the Cross" (Museo del Prado, Madrid) by the Nederlandish painter Rogier van der Weyden (1399/14001464). The aim was to address the question of to what extent oil and century egg tempera were used together as binding medium in 15 th century paintings. Imaging secondary ion mass spectrometry in positive and negative ion mode was performed using a $25 \mathrm{keV}{ }^{115} \mathrm{In}^{+}$primary ion beam. The total primary ion dose was $7.2 \times 10^{10}$ ions $/ \mathrm{cm}^{2}$. The surface of the sample was charge compensated with electron pulses in between the primary ion beam pulses. Fig. 1 shows the images obtained over an area of $250 \mu \mathrm{m} \times 250 \mu \mathrm{m}$. A total of six layers can be observed in the total positive $\left(\mathrm{TIC}^{+}\right)$and negative ion current $\left(\mathrm{TIC}^{-}\right.$) images. Images of sodium and aluminium could be correlated with the aid of optical microscopy to the blue pigment ultramarine $\left(\mathrm{Na}_{8-10} \mathrm{Al}_{6} \mathrm{Si}_{6} \mathrm{O}_{24} \mathrm{~S}_{2-4}\right)$, while copper, lead and calcium could, respectively be correlated to azurite particles $\left(2 \mathrm{CuCO}_{3} \cdot \mathrm{Cu}(\mathrm{OH})_{2}\right)$, lead white $\left(2 \mathrm{~Pb}\left(\mathrm{CO}_{3}\right)_{2} \cdot \mathrm{Pb}(\mathrm{OH})_{2}\right)$ which surrounds the azurite particles, and chalk $\left(\mathrm{CaCO}_{3}\right)$ as a ground layer. Organic fragments, such as $\mathrm{CO}_{3}$, deprotonated palmitic acid ( ${ }^{-} \mathrm{OOC}\left(\mathrm{CH}_{2}\right)_{14} \mathrm{CH}_{3}, \mathrm{~m} / \mathrm{z}$ $255)$ deprotonated stearic acid ( $\left.{ }^{-} \mathrm{OOC}\left(\mathrm{CH}_{2}\right)_{16} \mathrm{CH}_{3}, \mathrm{~m} / \mathrm{z}, 283\right)$ and palmitic acid soap $\left(\mathrm{Pb}^{2+}\left[{ }^{-} \mathrm{OOC}\left(\mathrm{CH}_{2}\right)_{14} \mathrm{CH}_{3}\right], \mathrm{m} / \mathrm{z} 460-\right.$ 463) are clearly present in the bottom three layers. They indicate the use of linseed oil as a binding medium. The presence of a fatty lead soap points furthermore to a mature oil containing medium. No evidence could be obtained for the presence of egg tempera [11].

In a separate study, the AMOLF research group has emphasised the importance of correct surface preparation for these imaging analytical studies, and developed a systematic dry polishing method whereby the sample is fixed in a specific polishing holder [13].

\section{Speciation of pigments}

The images also indicate the possibility of obtaining molecule specific information. SSIMS has emerged as a promising technique in this context [14]. Its potential advantage for obtaining molecule specific information lies primarily in the use of a low flux ion bombardment resulting in high relative molecular ion yields, although the total ion yield may be low. The latter problem can be resolved by using a mass analyser with high transmission, such as a time-of-flight mass spectrometer, therefore obtaining a good sensitivity. In addition, the use of polyatomic projectiles offers, in principle, significant potential to increase the molecular information in SSIMS. It has been demonstrated that bombardment of binary salts with polyatomic instead of atomic primary ions gives a substantial gain in the total ion current by a factor 4-10 depending on the 

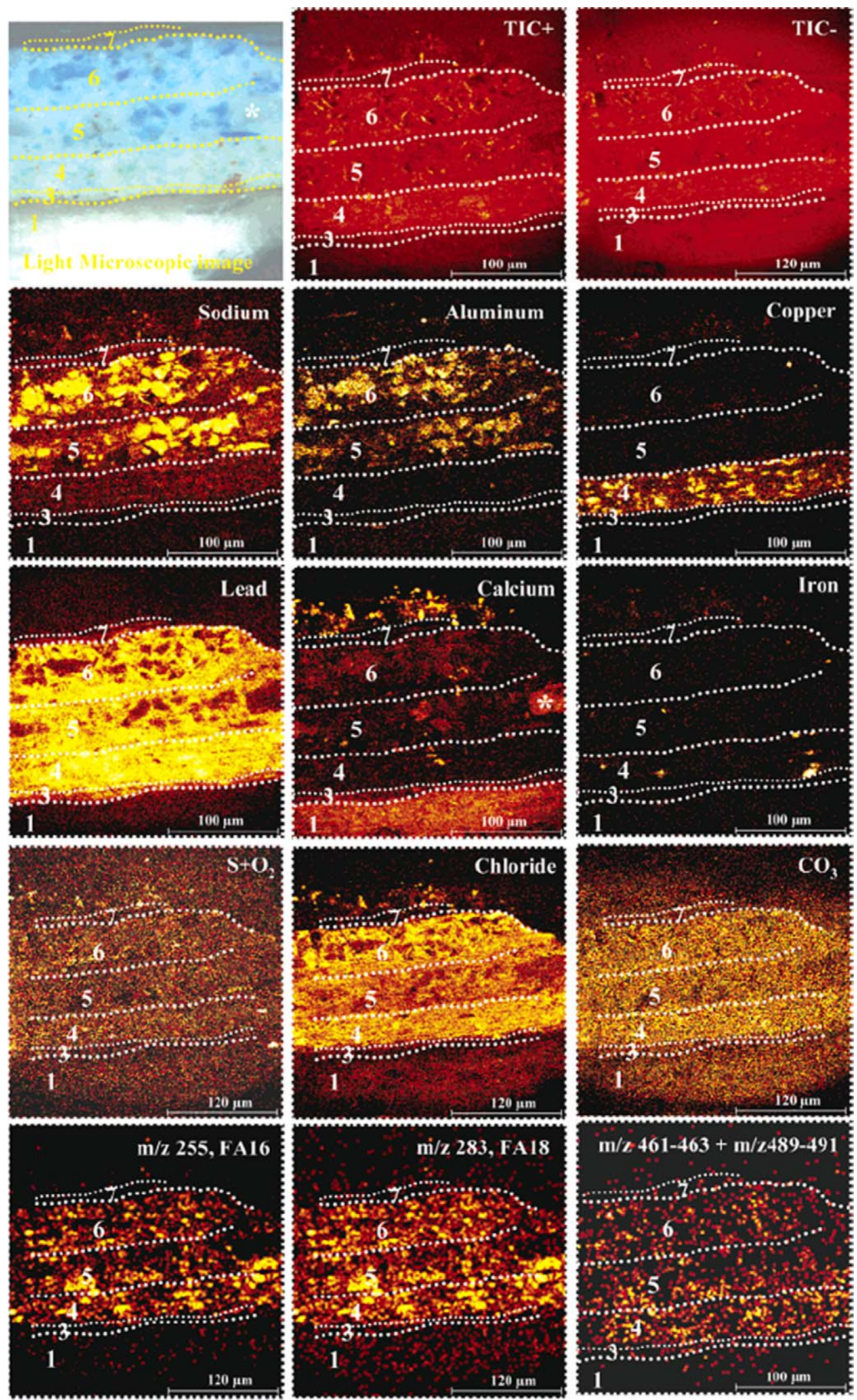

Fig. 1. Light micrograph (upper left) of the paint layer cross section and static SIMS images showing the spatial distribution of the total ion current (TIC ${ }^{+}$) and (TIC ${ }^{-}$), sodium, aluminium, copper, lead, calcium, iron, sulphur, chloride, carbonate, deprotonated palmitic acid $(\mathrm{m} / \mathrm{z} 255)$, stearic acid $(\mathrm{m} / \mathrm{z} / 283)$ and a sum image of palmitic acid and stearic acid lead soap $(\mathrm{m} / \mathrm{z}$ 461-463, 489-491). After [11]. 


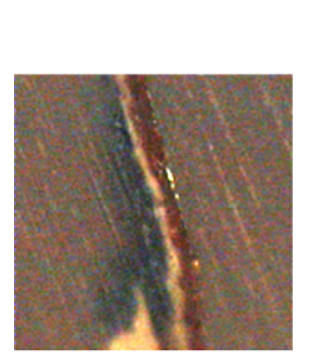

File: 1080301 SV.MIF

Field of view: $150 \times 150 \mu \mathrm{m}^{2}$

Scans: $1-17$ Pulses/Pixel: 32 $\longmapsto 20 \mu \mathrm{m}$
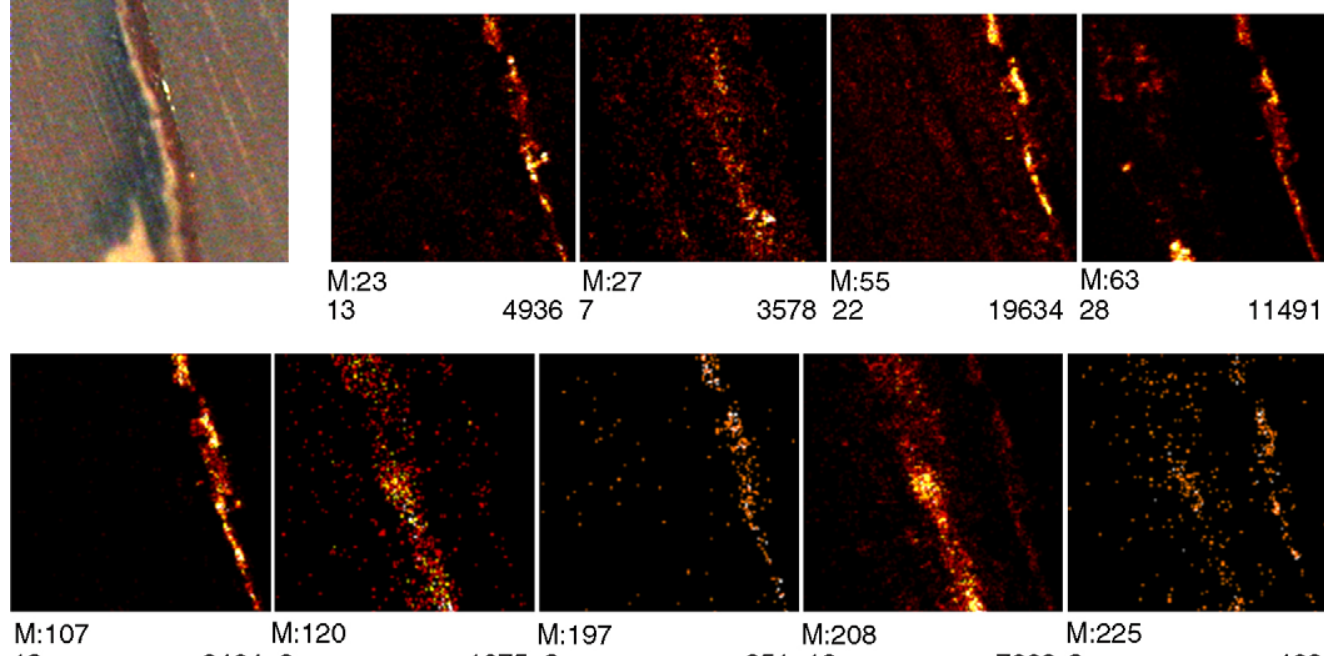

10752

35113

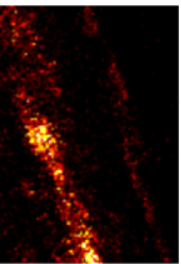

7668

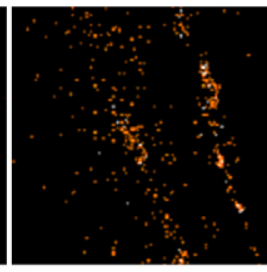

$M: 225$
M:197

2

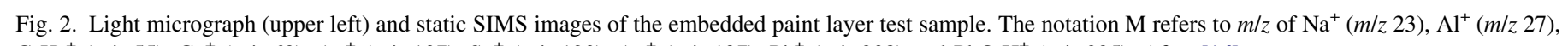
$\mathrm{C}_{3} \mathrm{H}_{5}{ }^{+}(m / z, 55), \mathrm{Cu}^{+}(m / z, 63), \mathrm{Ag}^{+}(m / z, 107), \mathrm{Sn}^{+}(m / z, 120), \mathrm{Au}^{+}(m / z, 197), \mathrm{Pb}^{+}(m / z, 208)$ and PbO$\cdot \mathrm{H}^{+}(m / z$ 225). After [16].

analogue under study, hence resulting in a substantial increase in the molecular specificity of the mass spectra [15].

In a feasibility study by Van Ham et al. [16] the possibilities of static SIMS were explored to characterise pure inorganic pigments used in paintings by means of molecular information. The main objective in the identification of pigments is to extract information about their historical origin for authenticity studies and the possible need for restoration. The characterization of a pigment can require different levels of sophistication, ranging from the identification of its colour to its chemical formula. In the latter case one may need to obtain molecule specific information as some pigments contain the same elements but have a different chemical formula. The study showed amongst others the possibility to differentiate verdigris, a copper acetate $\left(\left(\mathrm{CH}_{3} \mathrm{COO}\right)_{2} \mathrm{Cu} \cdot 2 \mathrm{Cu}(\mathrm{OH})_{2}\right)$ from another commonly used green pigment, malachite, a copper carbonate $\left(\mathrm{CuCO}_{3} \cdot \mathrm{Cu}(\mathrm{OH})_{2}\right)$.

The study was extended and involved the analysis of artificial layer samples which were prepared at the Kunsthistorisches Museum in Vienna. Pre-polymerised linseed oil was used as binding medium for the paint whereas the upper varnish layer consisted of mastic resin dissolved in oil of turpentine. The sample contains six layers: (1) gold foil; (2) cinnabar red $(\mathrm{HgS})$; (3) red iron oxide paint layer $\left(\mathrm{Fe}_{2} \mathrm{O}_{3} \cdot \mathrm{nH}_{2} \mathrm{O}\right)$; (4) lead-tin yellow paint layer $\left(\mathrm{Pb}_{2} \mathrm{SnO}_{4}\right)$; (5) azurite paint layer (2 $\left.\mathrm{CuCO}_{3} \cdot \mathrm{Cu}(\mathrm{OH})_{2}\right)$ and (6) varnish. Paint fragments of about $1 \mathrm{~mm}^{2}$ and $0.1 \mathrm{~mm}$ thick were embedded in resin and polished to expose the cross section at the surface.

Static SIMS imaging was performed with a $\mathrm{Ga}^{+}$primary ion beam mode with charge compensation. The raster area was $150 \mu \mathrm{m} \times 150 \mu \mathrm{m}$ and the analysis time was $890 \mathrm{~s}$ with a total ion dose of $2.5 \times 10^{12}$ ions $\mathrm{cm}^{-2}$. Fig. 2 shows the positive ion images of the sample together with the light microscopy image obtained with a camera in the SIMS instrument itself. Specifically, the gold layer is seen in the $m / z 197$ image, although $\mathrm{Ag}^{+}(\mathrm{m} / \mathrm{z}, 107)$ appears to be more abundantly generated from the same layer. The $\mathrm{Na}^{+}$and $\mathrm{Cu}^{+}$images show an enrichment of the metallic layer while also the organic ions at $\mathrm{m} / \mathrm{z}$ 55 are intensified. The next layer seems to be enriched with Al but no ions from the red $\mathrm{HgS}$ pigment are generated. The lead-tin yellow coating is seen from the $\mathrm{Sn}^{+}$and $\mathrm{Pb}^{+}$images. $\mathrm{As} \mathrm{Pb}_{2} \mathrm{SnO}_{4}$ consists of a mixture of $\mathrm{PbO}$ and $\mathrm{SnO}_{2}$, the $\mathrm{PbO} \cdot \mathrm{H}^{+}$ions represent direct molecular information on a specific component. Intense $\mathrm{Cu}^{+}$ions localise the blue azurite layer. Images of ions at higher $\mathrm{m} / \mathrm{z}$ do not contain significant information.

Only information from elemental ions was obtained from the layered samples, whereas the analysis of pure pigments such as auripigment and verdigris resulted in the detection of numerous high $\mathrm{m} / \mathrm{z}$ ions for molecular identification. The authors attribute this to the sample mounting method [16] which involved setting a small paint fragment in the middle of a relatively large and thick cube of non-conductive resin. The resulting charge buildup is significant and, in our experience, detrimental to the detection of high $\mathrm{m} / \mathrm{z}$ ions.

\section{Low-energy depth profiling of glass}

Depth profiling has been a common technique for several years in the study of the deterioration of glass objects [6-9,1722]. Investigations have shown that exposure to a moist environment causes the glass to deteriorate, through both chemical and structural changes. The initial stage of the attack is a process which involves ion exchange between the alkali ions which are present in the silicate structure of the glass, such as $\mathrm{Na}, \mathrm{K}$ and hydrogen from the environment [23]. These analyses were done on glass objects with thick corrosion crusts (several microns to even tens of micrometers). The latter means they were exposed to humid conditions for a very long time or were corroded artificially at an accelerated rate (i.e. high temperature). 
Advances in SIMS instrumentation over the past years have led to the possibility of carrying out depth profiling using sub$\mathrm{keV}$ beam energies [24], implying nanometre depth resolution. This means that glass corrosion can now be performed at room temperature, over reasonable time periods, and accurate depth concentration profiles of the leaching alkali ions can be obtained from the top $50-80 \mathrm{~nm}$. One example is the study by Fearn et al. [22] which focuses on obtaining a more precise description of the mechanism of glass corrosion at room temperature, in order to help museum conservators to select suitable environmental conditions for the safe storage of vulnerable glass objects. Replica glasses were produced of a particular composition, which were left for a period of time to age at room temperature. The samples were profiled with a nitrogen ion beam at $500 \mathrm{eV}$. The current was $90 \mathrm{nA}$ and the scan size $375 \mu \mathrm{m}$ square. Charge compensation was applied.

Fig. 3 shows typical concentration depth profiles for the main elemental species of the aged replica glass at $55 \% \mathrm{RH}$ for $91 \mathrm{~h}$ at room temperature. At the start of the SIMS profile both the $\mathrm{Na}$ and $\mathrm{K}$ signals drop to a minimum. This represents the removal of the leached corrosion products from the surface of the glass. After reaching a minimum, both signals then gradually increase to flat stable signals indicating that the bulk glass state has been reached. Based on a set of experiments with different levels of humidity (room temperature, $91 \mathrm{~h}$ ), the authors were able to prove that even for the very short period of exposure, the sample becomes very rapidly depleted in $\mathrm{Na}$ and K. From these early SIMS results they were able to conclude that alkali ions leach from the surface under storage conditions which have been suggested as safe.

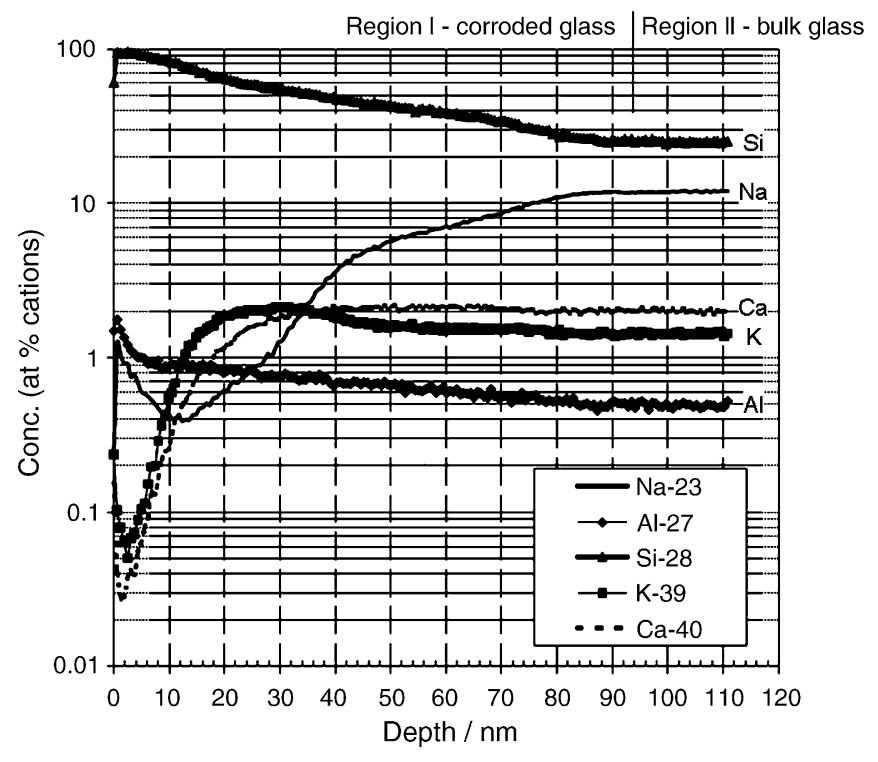

Fig. 3. Typical concentration depth profiles for the main elemental species of the aged replica glass. The concentration scale has been quantified by setting the bulk glass signals to the bulk concentration levels of the glass obtained via EPMA. The glass has been aged at $55 \% \mathrm{RH}$ for $91 \mathrm{~h}$ at room temperature. After [22].

\section{Ultra-low energy surface studies on metals}

The corrosion of ancient metal surfaces in storage and on display is a very serious problem in museums and historical sites, and it results in two questions: how to prevent the corrosion in the first place, and how to treat corroded surfaces. Whilst static SIMS can give some information, the real
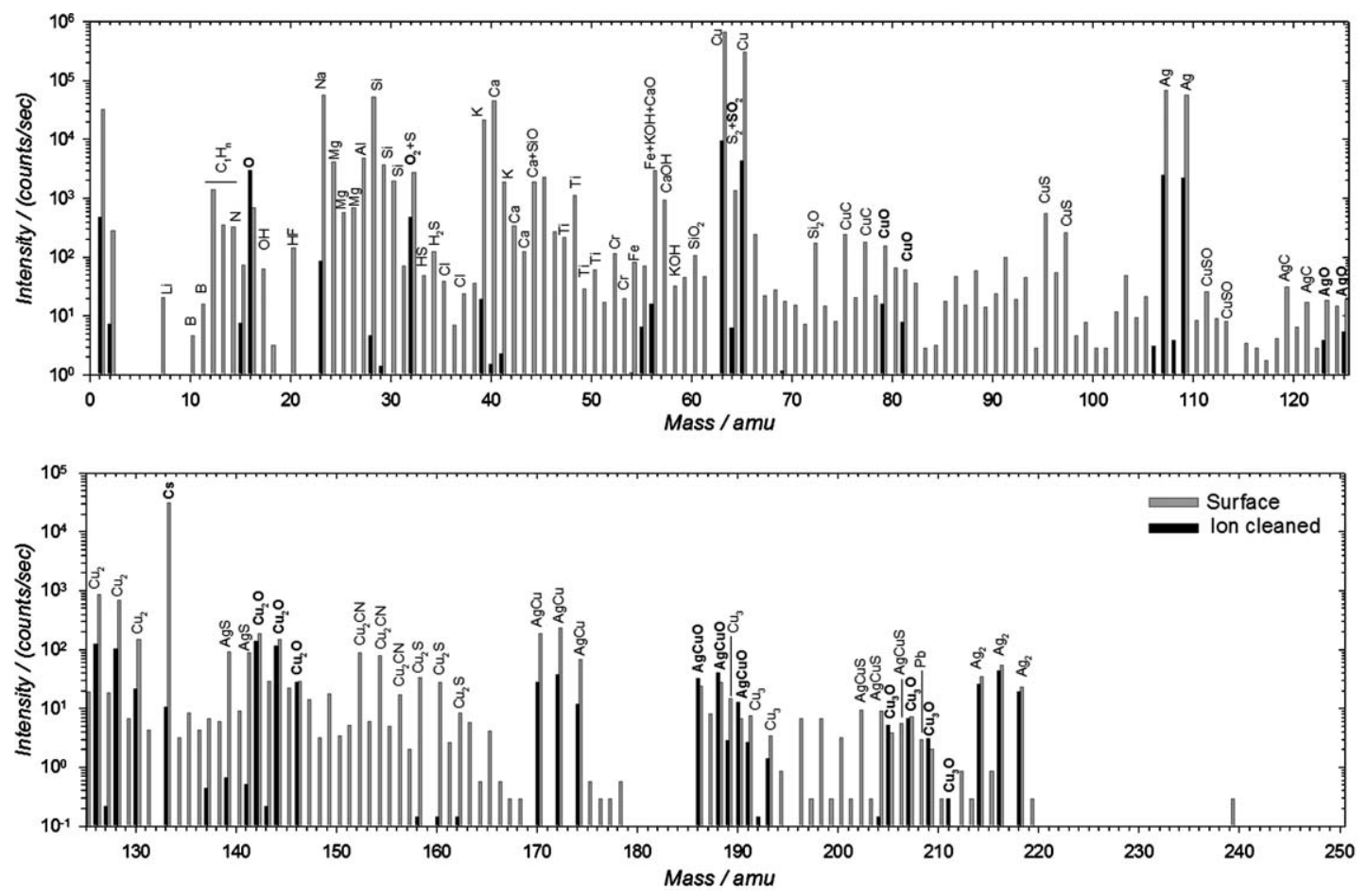

Fig. 4. Ultra-low energy dynamic SIMS: positive ion spectrum from the surface of a Sterling silver coupon polished with "Tripoli" and exposed in a glass cabinet for 2 years in the Swiss National Museum (grey bars) compared with the spectrum from bulk sterling silver (black bars). Primary ions: $10 \mathrm{nA}, 500 \mathrm{eV}^{16} \mathrm{O}_{2}{ }^{+}$incident at $30^{\circ}$ to normal and scanned over a $1 \mathrm{~mm}$ square (surface spectrum), and stationary beam $50 \mu \mathrm{m}$ diameter at the bottom of a $35 \mu \mathrm{m}$ deep crater (bulk spectrum). After [25]. 
corrosion may start some nanometres or more below a layer of atmospheric contamination and polish residue. We have found that $[25,26]$ even surfaces prepared with semiconductor grade chemicals (e.g. hyper pure water and organic solvents) and exposed to air under clean conditions, are coated with sufficient particulate and layered material to make the study of inorganic surface reactions a problem. Using ultra-low energy dynamic SIMS both as a depth profiling and a spectroscopy tool allows superficial nanometre scale layers to be cleaned off during the analysis. In a similar way to the glass study, surface corrosion at the very earliest stages may be monitored, and much information can be gained about residues left by polishing [25]. Fig. 4 shows a positive ion spectrum (light grey bars) taken with $10 \mathrm{nA} 500 \mathrm{eV} \mathrm{O}_{2}{ }^{+}$incident at $30^{\circ}$ with a $1 \mathrm{~mm}$ square scan on a sterling silver coupon polished with "Tripoli" (a proprietary polishing compound which is formulated to mimic the properties of crushed siliceous limestone). After polishing, the coupon was exposed for 2 years in a glass cabinet at the Swiss National Museum, and had a dark film of tarnish some nanometres thick. For comparison, the dark bars show a spectrum taken under similar conditions but using a stationary beam at the bottom of a crater $35 \mu \mathrm{m}$ deep to get a spectrum representative of the bulk material. (In the latter, the oxide signals mostly come from interaction with the beam.) Tripoli particles embedded in the surface contribute most of the metal signals between 20 and $60 \mathrm{Da}$. The negative ion spectrum (not shown) shows that the tarnish is largely a mixture of $\mathrm{Cu}$ and $\mathrm{Ag}$ sulphides; nevertheless cyanides and chlorides are also evident in both positive and negative ion spectra. The fingerprint spectra from the tarnish sputter away very rapidly, indicating that the layer is only a few nanometres thick. Particle signatures persist in the spectra for several microns into the silver.

\section{Conclusions}

This paper has discussed the capabilities and limitations of SIMS for a selection of very recent applications in the field of archaeometry and cultural heritage. Sample preparation is especially important, and inappropriate polishing techniques (for example) can completely change the data obtained, whether it be from a pigment, or a metal surface. When addressing such problems it should be kept in mind that a synergistic combination of techniques is needed, and these must be carefully matched to the problem. In the ideal case, the outcome from conservation and archaeometric research is an object conserved according to the state-of-the-art knowledge in scientific analysis and conservation techniques, complete with an information set giving origin, provenance, use, dating and manufacture in a form suitable for delivery to the professional inquirer, the public and for journal publication.

\section{References}

[1] A. Adriaens, Trends Anal. Chem. 23 (8) (2004) 583.

[2] A. Adriaens, G. Demortier, Nucl. Instrum. Methods B 226 (2004) 3.

[3] D. Creagh, D. Bradley (Eds.), Radiation in Art and Archaeometry, Elsevier Science B.V, Amsterdam, 2000.

[4] E. Ciliberto, G. Spoto (Eds.), Modern Analytical Methods in Art and Archaeology, Chemical Analysis, vol. 155, Wiley, New York, 2000.

[5] K. Janssens, R. Van Grieken (Eds.), Non-Destructive Microanalysis of Cultural Heritage Materials, Elsevier Science B.V., Amsterdam, 2004.

[6] A. Adriaens, in: D.C. Creagh, D.A. Bradley (Eds.), Radiation in Art and Archaeology, Elsevier, Amsterdam, The Netherlands, 2000, p. 397.

[7] G. Spoto, Thermochim. Acta 365 (1-2) (2000) 157.

[8] E. Darque-Ceretti, M. Aucouturier, in: K. Janssens, R. Van Grieken (Eds.), Non-destructive Microanalysis of Cultural Heritage Materials, Elsevier Science B.V., Amsterdam, 2004, p. 73.

[9] M. Dowsett, A. Adriaens, Nucl. Instrum. Methods B 226 (2004) 38.

[10] K. Keune, Binding medium, pigments and metal soaps characterized and localized in paint cross sections, dissertation, Molart report, Archetype, London, 2005.

[11] K. Keune, J. Boon, Anal. Chem. 76 (5) (2004) 1374.

[12] K. Keune, J. Boon, Anal. Chem. 77 (15) (2005) 4742.

[13] A. Van Loon, K. Keune, J. Boon, in: C. Parisi, G. Buzzanca, A. Paradisi (Eds.), Proceedings of the 8th International Conference on Non-Destructive Investigations and Microanalysis for the Diagnostics and Conservation of the Cultural and Environmental Heritage, Italian Society of Non Destructive Testing Monitoring Diagnostics, Rome, (2005), p. 89.

[14] R. Van Ham, L. Van Vaeck, F. Adams, A. Adriaens, Anal. Chem. 76 (9) (2004) 2609.

[15] R. Van Ham, L. Van Vaeck, A. Adriaens, F. Adams, B. Hodges, G. Groenewold, J. Anal. At. Spectrom. 17 (2002) 753.

[16] R. Van Ham, L. Van Vaeck, F. Adams, A. Adriaens, Anal. Bioanal. Chem. 383 (2005) 991.

[17] M. Schreiner, G. Stingeder, M. Grasserbauer, Fres. J. Anal. Chem. 319 (1984) 600.

[18] M. Schreiner, Mikrochim. Acta II (1991) 255.

[19] A. Aerts, Microscopic Analysis of Roman Vessel Glass, dissertation, University of Antwerp, Belgium, 1998.

[20] I. De Raedt, Composition of 16-17th century Facon-de-Venise glass excavated in Antwerp and neighbouring cities, dissertation, University of Antwerp, Belgium, 2002.

[21] J.L. Ryan, D.S. McPhail, P.S. Rogers, V.I. Oakley, in: Proceedings of the ICOM 11th triennial meeting, 1996, p. 839.

[22] S. Fearn, D.S. McPhail, V. Oakley, Appl. Surf. Sci. 231-232 (2004) 510.

[23] R.H. Doremus, Glass Science, Wiley, New York, 1973.

[24] M.G. Dowsett, Appl. Surf. Sci. 203-204 (2003) 5.

[25] M.G. Dowsett, A. Adriaens, M. Soares, H. Wouters, V.V.N. Palitsin, R. Gibbons, R.J.H. Morris, Nucl. Instrum. Methods B 239 (2005) 51.

[26] V.V. Palitsin, M.G. Dowsett, B. Guzmán de la Mata, I.W. Oloff, R. Gibbons, Appl. Surf. Sci., this issue. 